\title{
How Vascular Smooth Muscle Cells Face The Oxidative Stress In The Pulmonary Arterial Hypertension?
}

\author{
Dongqing Guo ${ }^{1 *}$, Shihong Jiao ${ }^{1}$ and Yuchun $\mathrm{Gu}^{2}$ \\ ${ }^{1}$ School of Life Sciences, Beijing University of Chinese Medicine, China \\ ${ }^{2}$ Aston Translational and Regenerative Medicine Centre, Aston Medical School, Aston University, UK
}

Submission: December 18, 2017; Published: January 08, 2018

*Corresponding author: Dongqing Guo, PhD, School of Life Sciences, Beijing University of Chinese Medicine, Beijing, China, 100029, Email: dong_qing_guo@126.com

\begin{abstract}
Oxidative stress occurs during the process of pulmonary arterial hypertension (PAH). PAH is a proliferative disorder associated with enhanced proliferation and suppressed apoptosis of pulmonary arterial smooth muscle cells. How the smooth muscle cells face the oxidative stress in the PAH? It has been reported that glycolytic enzyme pyruvate kinase M2 isoform (PKM2) could maintain cellular redox homeostasis during PAH. In this paper, we focus on the relationship between reactive oxygen species (ROS) and PKM2 in PAH.
\end{abstract}

Keywords: ROS, pulmonary arterial hypertension, PKM2

\section{Mini Review}

Pulmonary arterial hypertension (PAH), a severe disease caused by increased pulmonary vascular resistance and finally leads to right ventricular (RV) failure [1]. The occurrence of pulmonary arterial hypertension is related to many factors, such as cell abnormality, molecular, genetic factors and so on. PAH is a proliferative disorder associated with enhanced proliferation and suppressed apoptosis of pulmonary artery smooth muscle cells (PASMCs) [2]. In the process of PAH, the smooth muscle cells switch from the contractile phenotype to proliferative phenotype [3], and occur over-proliferation and hypertrophy, resulting in increased thickness of pulmonary artery. Markers of smooth muscle cell contraction phenotype such as SM22a, SM a-actin and calmodulin calponin, whose expressions all decreased in the PAH [4]. The imbalance of proliferation and apoptosis of pulmonary arterial smooth muscle cells leads to the increase of pulmonary vascular resistance and pulmonary arterial pressure.

Excessive levels of reactive oxygen species (ROS) and increased expression of NADPH oxidases (Nox) have been proposed to contribute to pulmonary artery hypertension $[2,5,6]$. Nox4 and Nox2-derived ROS increase can trigger pulmonary vascular remodeling induced by hypoxia [7]. In contrast to hypoxia-induced $\mathrm{PAH}$, Nox1 is responsible for pathophysiological proliferation and migration of PASMC in MCT- induced PAH via increased superoxide production [8]. It is well known that ROS over-accumulation results in significant damage to cell structure. However, relatively little is known about how the RPASMCs tolerate the increasing oxidative stress and overproliferate.

Most cells utilize glucose as energy source to produce ATP. The formation of pyruvate by multistep glycolysis enters the mitochondria, which is oxidized through the tricarboxylic acid cycle and produces ATP to meet the energy requirements for the cells [9]. However, in cancer cells or other highly proliferative cells, most pyruvate produced by glycolysis does not enter the mitochondria and instead to produce lactate in the presence of lactate dehydrogenase (LDH). Lactate generally produced in hypoxia, but cancer cells will give priority to use glycolysis to produce ATP even in sufficient oxygen, which is proposed in early 1920s by Otto Warburg. We call this phenomenon as "Warburg effect" or "aerobic glycolysis" [10]. In Guo 's paper [11], isolated pulmonary arterial smooth muscle cells were treated with different concentration of 2-DG and oligomycin. 2-DG is an inhibitor of glycolysis and oligomycin is the inhibitor of mitochondrial oxidative phosphorylation. As a result, 2-DG obviously inhibited intracellular ATP production of RPASMC and suggested that glycolysis was the dominant metabolic way for RPASMC. 
Pyruvate kinase (PK) is the key rate-limiting enzyme involved in glycolysis. It catalyzes phosphoenolpyruvate (PEP) to generate pyruvate [12]. There are four isoforms of PK in mammals including PKL, PKR, PKM1 and PKM2 [13]. Among them, the M1 is expressed in almost all adult tissues; and the M2 splice variant is expressed during embryonic development, in undifferentiated tissues, and in tumors $[12,14,15]$. It has been reported that M2 isoform of pyruvate kinase (PKM2) is preferentially expressed in cancer cells and is a central point of regulator in cancer metabolism [16]. PKM2 may confer an additional advantage to cancer cells by allowing them to withstand oxidative stress [17]. RPASMC are very similar with cancer cells, which have suppressed apoptosis, enhanced proliferation and migration in the pathogenesis of PAH. Thus PKM2 may function in the process of PAH (Figure 1).

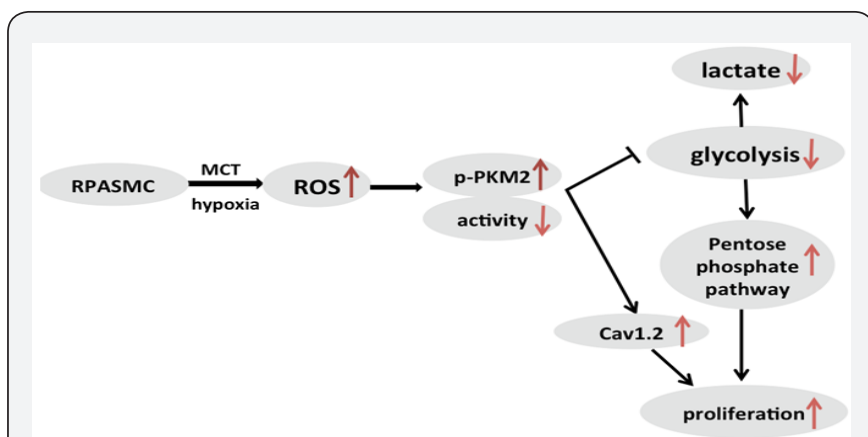

Figure 1: Schematic illustrating the signaling mechanisms triggered by ROS to promote cells proliferation in $\mathrm{PAH}$.

Guo et al. [11] showed that ROS accumulation in the smooth muscle cells led to the inhibition of PKM2 activity in the pulmonary hypertension. PKM2 activity decreased while the phosphorylation of PKM2 increased. The ROS inhibitor including NAC, apocynin and MnTBAP could reverse the effects in PAH. Then they explored the mechanism that inhibition of PKM2 activity results in the cell proliferation. Decreased PKM2 activity modulated the flux of glycolytic intermediates in support of cell proliferation through the increased pentose phosphate pathway (PPP). The pentose phosphate pathway is a metabolic pathway parallel to glycolysis. It generates NADPH and pentoses, a precursor for the synthesis of nucleotides [18]. The pentose phosphate pathway is related to the cell proliferation. Preter $G$ has demonstrated that inhibition of glucose-6-phosphate dehydrogenase, the first and rate-limiting enzyme of the PPP could decrease cancer cells proliferation [19]. What's more, the decrease PKM2 activity promoted the expression of Cav1.2 which also could promote cell proliferation [20].

\section{Conclusion}

In $\mathrm{PAH}$, the key glycolytic enzyme PKM2 involves in maintaining cellular redox homeostasis in a negative feedback loop. Increasing in cellular ROS promotes the phosphorylation of PKM2, and inactivates its catalytic activity, which promotes glucose to enter the pentose phosphate pathway and cell proliferation. PKM2 activators may provide a new target in the treatment of PAH.

\section{Acknowledgements}

This work is supported by National Natural Science Foundation of China held by DG (No.81703836).

\section{References}

1. McLaughlin VV, Archer SL, Badesch DB Barst RJ, Farber HW, et al. (2009) ACCF/AHA 2009 Expert consensus document on pulmonary hypertension a report of the American College of Cardiology Foundation Task Force on Expert Consensus Documents and the American Heart Association developed in collaboration with the American College of Chest Physicians; American Thoracic Society, Inc.; and the Pulmonary Hypertension Association. J Am Coll Cardiol 53(17): 1573-1619.

2. Sanders KA, Hoidal JR (2007) The NOX on pulmonary hypertension. Circulation Research 101: 224-226.

3. Song Z, Li G (2010) Role of specific microRNAs in regulation of vascular smooth muscle cell differentiation and the response to injury. J Cardiovasc Transl Res 3(3): 246-250.

4. Zhang J, Hu H, Palma NL Harrison JK, Mubarak KK, et al. (2012) Hypoxia-induced endothelial CX3CL1 triggers lung smooth muscle cell phenotypic switching and proliferative expansion. Am J Physiol Lung Cell Mol Physiol 303: L912-922.

5. Touyz RM (2004) Reactive oxygen species, vascular oxidative stress, and redox signaling in hypertension - What is the clinical significance? Hypertension 44(3): 248-252.

6. Frazziano G, Al Ghouleh I, Baust J, Shiva S, Champion HC, et al. (2014) Nox-derived ROS are acutely activated in pressure overload pulmonary hypertension: indications for a seminal role for mitochondrial Nox4. Am J Physiol Heart Circ Physiol 306: H197-205.

7. Ismail S, Sturrock A, Wu P, Cahill B, Norman K, et al. (2009) NOX4 mediates hypoxia-induced proliferation of human pulmonary artery smooth muscle cells: the role of autocrine production of transforming growth factor-\{beta\} 1 and insulin-like growth factor binding protein-3. Am J Physiol Lung Cell Mol Physiol 296(3): L489-499.

8. Veit F, Pak O, Egemnazarov B, Roth M, Kosanovic D, et al. (2013) Function of NADPH Oxidase 1 in Pulmonary Arterial Smooth Muscle Cells After Monocrotaline-Induced Pulmonary Vascular Remodeling. Antioxidants \& Redox Signaling 19(18): 2213-2231.

9. Kefas B, Comeau L, Erdle N, Montgomery E, Amos S, et al. (2010) Pyruvate kinase M2 is a target of the tumor-suppressive microRNA-326 and regulates the survival of glioma cells. Neuro Oncol 12(11): 11021112.

10. Hamanaka RB, Chandel NS (2011) Warburg Effect and Redox Balance. Science 334(6060): 1219-1220.

11. Guo DQ Gu JZ, Jiang H, Ahmed A, Zhang Z, et al. (2016) Inhibition of pyruvate kinase $\mathrm{M} 2$ by reactive oxygen species contributes to the development of pulmonary arterial hypertension. Journal of Molecular and Cellular Cardiology 91: 179-187.

12. Mazurek S, Boschek CB, Hugo F, Eigenbrodt E (2005) Pyruvate kinase type $\mathrm{M} 2$ and its role in tumor growth and spreading. Semin Cancer Biol. 15(4): 300-308.

13. Cortes-Cros M, Hemmerlin C, Ferretti S, Zhang J, Gounarides JS, et al. (2013) M2 isoform of pyruvate kinase is dispensable for tumor maintenance and growth. Proc Natl Acad Sci U S A 110(2): 489-494.

14. Dombrauckas JD, Santarsiero BD, Mesecar AD (2005) Structural basis for tumor pyruvate kinase M2 allosteric regulation and catalysis. Biochemistry 44(27): 9417-9429. 
15. Christofk HR, Vander Heiden MG, Harris MH, Ramanathan A, Gerszten $\mathrm{RE}$, et al. (2008) The M2 splice isoform of pyruvate kinase is important for cancer metabolism and tumour growth. Nature 452(7184): 230 233

16. Li J, Yang Z, Zou Q, Yuan Y, Li J, et al. (2014) PKM2 and ACVR 1C are prognostic markers for poor prognosis of gallbladder cancer. Clin Transl Oncol 16(2): 200-207.

17. Anastasiou D, Poulogiannis G, Asara JM, Boxer MB, Jiang JK et al. (2011) Inhibition of pyruvate kinase $\mathrm{M} 2$ by reactive oxygen species contributes to cellular antioxidant responses. Science 334(6060): 1278-1283.
18. Wasylenko TM, Ahn WS, Stephanopoulos G (2015) The oxidative pentose phosphate pathway is the primary source of NADPH for lipid overproduction from glucose in Yarrowia lipolytica. Metab Eng 30: 27 39.

19. De Preter G, Neveu MA, Danhier P, Brisson L, Payen VL et al. (2016) Inhibition of the pentose phosphate pathway by dichloroacetate unravels a missing link between aerobic glycolysis and cancer cell proliferation. Oncotarget 7(3): 2910-2920

20. Soldatov NM (2013) Cav1.2, cell proliferation, and new target in atherosclerosis. ISRN Biochem 2013: 463527. 